\title{
Progress in coagulation rate measurements of colloidal dispersions
}

\author{
Shenghua $\mathrm{Xu}^{a b}$ and Zhiwei Sun $* a b$ \\ Received 1st July 2011, Accepted 31st August 2011 \\ DOI: 10.1039/c1sm06237a
}

This article reviews recent advances in coagulation rate measurements of colloidal dispersions, with emphasis on the turbidity method. For turbidity method, measurement of the coagulation rate relies upon the turbidity change resulting from the coagulation process, and the measuring sensitivity significantly depends on particle size and the wavelength used. There exists a "zero sensitivity" blind point for measurement at a specific wavelength, suggesting that such measurements should be performed at a wavelength some distance from the blind point. The major difficulty in determining absolute coagulation rate constant (CRC) by light scattering and turbidity measurements is how to theoretically solve the scattering problem of 2-particle aggregates. The T-matrix method accurately solves this problem, showing its superiority over various earlier theoretical approximations (applicable only to small particles). Results from studies on effects of forward scattering, multiple scattering, etc., provide guidelines for choosing proper particle size and volume fraction for the allowed margin of measurement error. Most of these findings on turbidity methods are also valid or applicable to other scattering methods. Finally, we introduce a new microscopic approach to assess the colloidal stability at individual particle levels, by means of directly observing artificially induced collision with the aid of optical tweezers.

${ }^{a}$ Key Laboratory of Microgravity, Institute of Mechanics, Chinese Academy of Sciences, Beijing, 100190, P. R. China. E-mail: sunzw@ imech.ac.cn

${ }^{b}$ National Microgravity Laboratory, Institute of Mechanics, Chinese Academy of Sciences, Beijing, 100190, P. R. China

\section{Introduction}

One of the core aspects of colloid science is investigating colloidal stability and coagulation kinetics. It is thus said that the subject matter of colloid science can be organized around the idea of

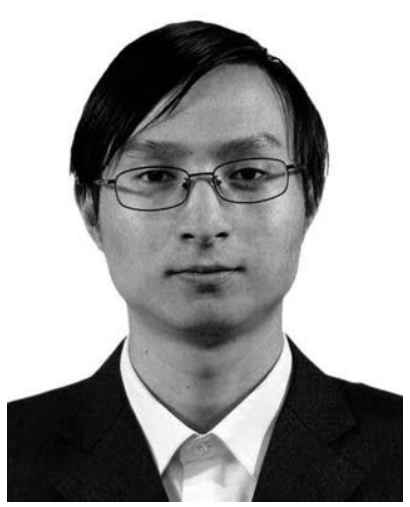

Shenghua Xu
Shenghua $\mathrm{Xu}$ is an Associate Professor of the Microgravity Lab at the Institute of Mechanics of the Chinese Academy of Sciences. He received his $P h D$ degree from the Department of Physics, University of Science and Technology of China. His current research interests include colloidal aggregation, phase transition in colloids, optical measuring technology, nonequilibrium interface \& surface dynamics.

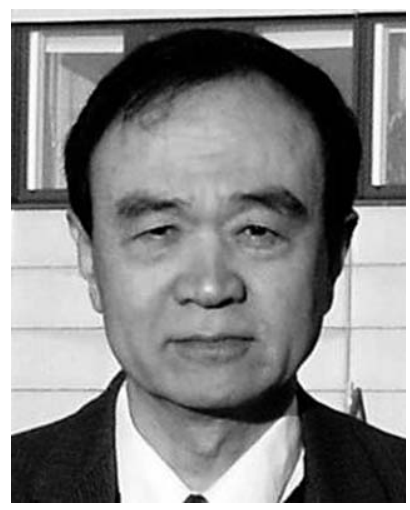

Zhiwei Sun
Zhiwei Sun is Professor of the Institute of Mechanics of the Chinese Academy of Sciences; adjunct Professor at Physics Department at the University of Science and Technology of China. He is periodically a visiting scholar at the Chemistry Department, University of California at Berkeley. Current research interests include colloidal aggregation, orderdisorder transition in colloidal suspensions, non-equilibrium interface dynamics, quantum Monte Carlo (QMC) method. As Senior Associate (1983-1993), worked in molecular electronic structure, specializing in QMC; modeling for surfactant micelles in aqueous solution at the Chemistry Department of UC-Berkeley, and the Materials Sciences Division of the Lawrence Berkeley National Laboratory, USA. 
stability. ${ }^{1}$ All the characteristics of colloidal systems change remarkably in the transition from the dispersed state to the aggregated state. Even within aggregated systems, the degree of aggregation varies tremendously. The coagulation rate constant (CRC) is an important parameter for characterizing the stability and coagulation kinetics of colloidal systems, enabling one to efficiently manipulate the states of dispersion for particular applications.

Experimental determination of the coagulation rate is required for various purposes, ranging from purely theoretical to very practical. Absolute CRCs are usually needed for comparing the theoretical predictions of aggregation kinetics with the experimental results, or for deriving information on interactions between particles. ${ }^{2-5}$ In many applications, on the other hand, the relative coagulation rates are sufficient..$^{5-7}$

There are basically two different approaches to measuring coagulation rate; namely, direct and indirect measurements. In direct measurement, ${ }^{1,5}$ particles are directly counted, and different particle sizes can be distinguished by employing various technical means. For indirect measurements, ${ }^{1,5}$ the coagulation rate is achieved by measuring some suspension property changes which occur due to the coagulation of particles. In this article, instead of discussing all methods of determining coagulation rate, we shall concentrate only on some new developments of this topic.

The following portions of this paper are organized as follows: Section 2 presents a brief overview of light scattering; Section 3 outlines recent progress relating to turbidity methods; Section 4 provides additional comments on light scattering methods. Section 5 introduces a new microscopic approach for evaluating colloidal stability. Finally, a summary is given in Section 6.

\section{Light scattering methods}

Since discussion of various light scattering techniques has been given in the literature, ${ }^{5,7-10}$ we present here only a brief overview.

To discuss the coagulation rate, we start with the simplest case of a dispersion, initially consisting of identical spherical particles.

At the very early stage of the coagulation process, only collisions of single particles which form doublets need to be considered. Therefore, the change of particle number concentrations can be approximately expressed as: $:^{11,12}$

$$
\begin{gathered}
\left(\frac{\mathrm{d} N_{1}}{\mathrm{~d} t}\right)_{t=0}=-k_{11} N_{1}{ }^{2} \\
\left(\frac{\mathrm{d} N_{2}}{\mathrm{~d} t}\right)_{t=0}=\frac{k_{11} N_{1}{ }^{2}}{2}
\end{gathered}
$$

where $N_{1}$ and $N_{2}$ are the number concentration of single particles and doublets, $t$ is time and $k_{11}$ is the CRC.

When light beam $I_{0}$ is passing through a colloidal suspension, a portion of the light will be scattered in all directions (see Fig. 1). The characteristics of the light scattered by the particles depend on their size, shape, refractive index, the light wavelength, the detection angle, etc. ${ }^{13}$ Various light-scattering methods for measuring the coagulation rate, including turbidimetry, are based on probing changes in characteristics of the light scattered by particles as a result of the coagulation.

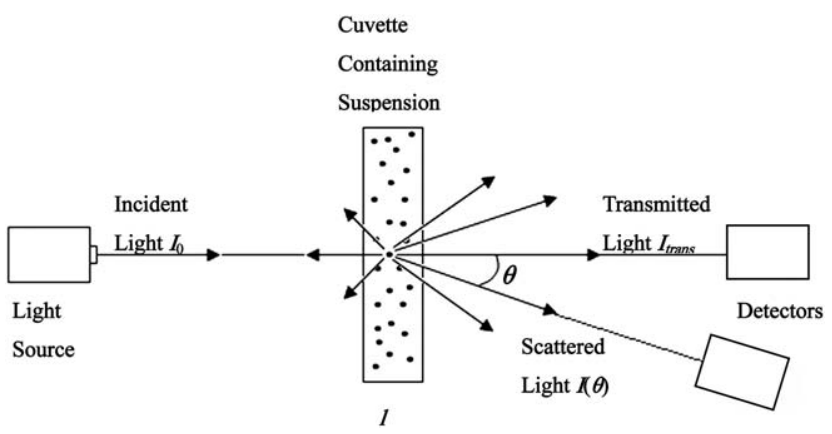

Fig. 1 Measurement of transmitted and scattered light. The distribution of scattered light by single particle is schematically shown by the length of the arrows.

\subsection{Static light scattering}

At the early stage of aggregation, the total scattering intensity $I$ $(q, t)$ at scattering vector $q$ at time $t$ can be written as: ${ }^{8}$

$$
I(q, t)=N_{1} I_{1}(q)+N_{2} I_{2}(q)
$$

where $I_{1}(q)$ and $I_{2}(q)$ are the scattered intensity of single particles and doublets, and $q=(4 \pi / \lambda) \sin (\theta / 2)$ in which $\lambda$ is the wavelength of incident light and $\theta$ is the scattering angle. Therefore,

$$
\frac{\mathrm{d} I(q, t)}{\mathrm{d} t}=\frac{\mathrm{d} N_{1}}{\mathrm{~d} t} I_{1}(q)+\frac{\mathrm{d} N_{2}}{\mathrm{~d} t} I_{2}(q)
$$

After inserting eqn (1) and (2), eqn (4) becomes:

$$
[\mathrm{d} I(q, t) / \mathrm{d} t]_{0}=-k_{11} N_{1}^{2} I_{1}(q)+k_{11} N_{1}^{2} I_{2}(q) / 2
$$

As a consequence,

$$
k_{11}=\frac{[\mathrm{d} I(q, t) / \mathrm{d} t]_{0} / I(q, 0)}{\left[I_{2}(q) / 2 I_{1}(q)-1\right] N_{1}}
$$

where $I(q, 0)$ is the total scattering intensity at $t=0$, and $I(q, 0)=$ $N_{1} I_{1}(q)$ because there are only single particles in the system. ${ }^{8}$

In order to calculate $k_{11}$ using eqn (6), the value of $I_{2}(q)$ and $I_{1}(q)$ are also needed. Since the light scattering properties of spherical particles and doublets are determined by the relative refractive index (the refractive index of scattering particles relative to the medium, which has nonzero imaginary part when there is absorption) and size parameter $\alpha=2 \pi a / \lambda, I_{2}(q)$ and $I_{1}(q)$ can be evaluated from theory when these parameters are known. ${ }^{8,13,14}$

A classical approximation for dealing with the scattering problems of particles is the Rayleigh-Gans-Debye (RGD) theory. ${ }^{13,15}$ As it is developed from Rayleigh theory, the RGD theory is only valid for small particles. In the study of colloidal coagulation, the colloidal suspension is usually dilute, so that the structure factor $S(q)$ can be neglected. ${ }^{16}$ The instrument resolution function is also generally ignored. ${ }^{8,14,17}$ Therefore, by using the RGD theory, the scattered intensity of aggregates can be expressed as: ${ }^{8}$

$$
I_{i}(q) \propto V_{i}^{2} P_{i}(q)
$$

where $V_{i}$ is the volume of aggregate of $i$ primary particles, and $P_{i}(q)$ is the so-called form factor, ${ }^{14,17,18}$ which is equal to 
$I_{i}(q) / I_{i}(0)$. By using RGD approximation, the form factor of the $i$-fold aggregate has the form: ${ }^{14}$

$$
P_{i}(q)=\frac{P_{1}(q)}{i^{2}}\left[\sum_{i, j} \frac{\sin \left(r_{\mu \nu} q\right)}{\left(r_{\mu \nu} q\right)}\right]
$$

where $r_{\mu \nu}$ is the distance of particle $\mu$ and $\nu$ in the aggregate, and $P_{1}(q)$ is:

$$
P_{1}(q)=\frac{9}{(q a)^{6}}[\sin (q a)-q a \cos (q a)]^{2}
$$

From eqn (8) and (9), using RGD approximation, ${ }^{13,15}$ one can find that $\left[I_{2}(q) / 2 I_{1}(q)-1\right]$ is approximately equal to $[\sin (2 a q) / 2 a q]$. Therefore, eqn (6) can then be expressed as:

$$
k_{11}=\frac{[\mathrm{d} I(q, t) / \mathrm{d} t]_{0} / I(q, 0)}{[\sin (2 a q) / 2 a q] N_{1}}
$$

where $a$ is the radius of a primary particle. $\operatorname{Eqn}(10)$ can then be used to evaluate the CRC $k_{11}$ after quantity $[\mathrm{d} I(q, t) / \mathrm{d} t]_{0} / I(q, 0)$ is measured by static light scattering method at single $q$ (or $\theta$ ).

To improve the accuracy of measurement, multi-angle light scattering has been adopted (see the literature ${ }^{19}$ ), in which the fitted lines of $[\mathrm{d} I(q, t) / \mathrm{d} t]_{0} / I(q, 0) \sim \sin (2 a q) / 2 a q$ are used to determine $k_{11}$.

For further simplification, small-angle light scattering is proposed in measuring the coagulation rate. ${ }^{20,21}$ In this case, the value of $q$ is close to 0 , so that the term $[\sin (2 a q) / 2 a q]$ in eqn (10) is approximately 1 .

\subsection{Dynamic light scattering}

In dynamic light scattering (DLS), ${ }^{22,23}$ also known as photon correlation spectroscopy, particle size is linked to Brownian motion and Doppler shift of light scattered by particles. The photon counter can be used to detect the scattered light, and it measures the fluctuations in light intensity due to the Doppler shift; this shift is related to particle sizes, because their diffusion coefficients are different. The particle coagulation causes changes in particle sizes, and the change of the average hydrodynamic radius can be used to evaluate $k_{11}$.

At the early stage of the aggregation, the average of the diffusion coefficient $\bar{D}$ measured by dynamic light scattering can be expressed as: ${ }^{8}$

$$
\bar{D}=\frac{N_{1} I_{1}(q) D_{1}+N_{2} I_{2}(q) D_{2}}{N_{1} I_{1}(q)+N_{2} I_{2}(q)}
$$

where $D_{1}$ and $D_{2}$ are the diffusion coefficients for single particle and doublet, respectively.

According to the Stokes-Einstein equation, the hydrodynamic radius of the particle $r_{\mathrm{h}}$ is related to the diffusion coefficient as: ${ }^{\mathbf{8 1 8}}$

$$
r_{\mathrm{h}}=\frac{k_{\mathrm{B}} T}{6 \pi \eta D}
$$

where $k_{\mathrm{B}}$ is the Boltzmann constant, $T$ is the temperature, and $\eta$ is the viscosity coefficient.

Then, eqn (11) can be rewritten as: ${ }^{8}$

$$
\frac{1}{r_{\mathrm{h}}(t)}=\frac{N_{1} I_{1}(q) / r_{\mathrm{h}, 1}+N_{2} I_{2}(q) / r_{\mathrm{h}, 2}}{N_{1} I_{1}(q)+N_{2} I_{2}(q)}
$$

where $r_{\mathrm{h}, 1}$ and $r_{\mathrm{h}, 2}$ are the hydrodynamic radii for single particle and doublet, respectively, and $r_{\mathrm{h}}(t)$ is the average hydrodynamic radius at time $t$.

Therefore,

$$
\begin{aligned}
\frac{r_{\mathrm{h}}(t)}{r_{\mathrm{h}}(0)} & =\frac{N_{1} I_{1}(q)+N_{2} I_{2}(q)}{N_{1} I_{1}(q)+N_{2} I_{2}(q)\left(r_{\mathrm{h}, 1} / r_{\mathrm{h}, 2}\right)} \\
& =1+\left(1-\frac{r_{\mathrm{h}, 1}}{r_{\mathrm{h}, 2}}\right) \frac{I_{2}(q)}{I_{1}(q)} \frac{N_{2}}{N_{1}+N_{2}\left(\frac{I_{2}(q) r_{\mathrm{h}, 1}}{I_{1}(q) r_{\mathrm{h}, 2}}\right)}
\end{aligned}
$$

Here $r_{\mathrm{h}}(0)=r_{\mathrm{h}, 1}$, because only primary particles exist at the beginning of aggregation. Considering $N_{2}=0$ at $t=0$ and eqn (2), after differentiation of eqn (14) with respect to $t$, one can get: ${ }^{8}$

$$
k_{11}=\frac{\left[\mathrm{d}\left(r_{\mathrm{h}}(t) / r_{\mathrm{h}}(0)\right) / \mathrm{d} t\right]_{0}}{\left(1-r_{\mathrm{h}, 1} / r_{\mathrm{h}, 2}\right)\left[I_{2}(q) / 2 I_{1}(q)\right] N_{1}}
$$

Similar to the static light scattering method, when RGD approximation is applicable, eqn (15) becomes:

$$
k_{11}=\frac{\left[\mathrm{d}\left(r_{\mathrm{h}}(t) / r_{\mathrm{h}}(0)\right) / \mathrm{d} t\right]_{0}}{\left(1-r_{\mathrm{h}, 1} / r_{\mathrm{h}, 2}\right)[\sin (2 a q) / 2 a q+1] N_{1}}
$$

The hydrodynamic factor $\left(1-r_{\mathrm{h}, 1} / r_{\mathrm{h}, 2}\right)$ is also needed to get the coagulation rate constant $k_{11}$ from eqn (16). Calculated from the hydrodynamic forces of two spheres in a low Reynolds number fluid, ${ }^{8,14,24}$ the relationship of $r_{\mathrm{h}, 1}$ and $r_{\mathrm{h}, 2}$ is $r_{\mathrm{h}, 2} \cong$ $1.38 r_{\mathrm{h}, 1}$, as is shown in ref. 8 . If the two spheres are free to rotate, then $r_{\mathrm{h}, 2} \cong 1.35 r_{\mathrm{h}, 1}{ }^{8}$

\subsection{Simultaneous static and dynamic light scattering (SSDLS)}

It can be seen that both static and dynamic light scattering need the evaluation of a quantity of $I_{2}(q) / 2 I_{1}(q)$, which can be done by using RGD approximation, but that can be difficult for largesized particles when RGD approximation is not applicable. To bypass this problem, Holthoff et al. ${ }^{8}$ suggested an approach in which eqn (6) and (15) are combined so that $I_{2}(q) / 2 I_{1}(q)$ is cancelled out. Therefore:

$$
k_{1} k_{11}=\left\{\left[\mathrm{d}\left(r_{\mathrm{h}}(t) / r_{\mathrm{h}}(0)\right) / \mathrm{d} t\right]_{0} /\left[1-r_{\mathrm{h}, 1} / r_{\mathrm{h}, 2}\right]-[\mathrm{d} I(q, t) / \mathrm{d} t]_{0} / I(q, 0)\right\} /
$$

Now, eqn (17) is not restricted only to small particles, because there is no need to calculate the quantity of $I_{2}(q) / 2 I_{1}(q)$ by appealing to RGD or other approximations in the measurement of the absolute coagulation rate..$^{25-28}$

\subsection{Improved approaches to calculating the form factor}

Since the RGD approximation is no longer applicable for large particles, several different theoretical approaches have recently been tried in order to 'lift' the restriction on particle size. These theoretical methods include the discrete dipole approximation (DDA), ${ }^{29}$ modal analysis (MA) ${ }^{14,30}$ and the T-matrix method. ${ }^{31-34}$ The capabilities of DDA and MA in dealing with large particles were verified in $\mathrm{CRC}$ measurements by comparing the form factors calculated using DDA and MA methods with those resulting from the SSDLS method. ${ }^{14}$

The T-matrix method ${ }^{31-34}$ has great capabilities for accurately computing electromagnetic scattering by single and compounded 
spherical particles, without limits of size or shape. Galletto et al. ${ }^{17}$ have compared the experimental form factors with calculations derived respectively by the T-matrix method and RDG approximation. Their measurements of form factors based on the SSDLS method were performed on dilute aggregating aqueous suspensions comprising asymmetric particle doublets composed of oppositely charged polystyrene latex particles. They found that the RDG approximation is reliable only up to particle diameters of about $250 \mathrm{~nm}$, while the T-matrix method is very accurate for all types of doublets which they investigated. Their results confirmed the suitability of the T-matrix method for accurately estimating the optical properties of colloidal particles in the micrometre range. As a highly efficient and cost-effective approach, the T-matrix technique has been used in determining absolute CRC by light scattering. ${ }^{10,17}$

\section{Progress in turbidity method}

\subsection{Turbidity method}

Turbidity is a measure of light loss of the transmitted beam caused by the scattering effect of particles. (For simplicity sake, zero absorbance is assumed here). Therefore, the information on particle aggregation can be extracted from the change in turbidity, which is associated with the change in characteristics of the light scattered by particles due to the coagulation.

Turbidity measurement of the coagulation rate has been extensively adopted ${ }^{5,35}$ because of its simplicity and ease of implementation.

If a monochromatic incident light beam of intensity $I_{0}$ traverses a suspension of length $(l)$ without any absorption, and its transmitted intensity becomes $I_{\text {trans, }}$, then the suspension's turbidity $(\tau)$ can be expressed as: ${ }^{12}$

$$
\tau=(1 / l) \ln \left(I_{0} / I_{\text {trans }}\right)
$$

At the early stage of the aggregation, only single particles and doublets exist, and the turbidity can be expressed as $\tau=N_{1} C_{1}+N_{2} C_{2}$, where $C_{1}$ and $C_{2}$ are the extinction (or attenuation) cross sections for single particle and doublet, respectively, and $\tau$ is the turbidity. ${ }^{12,35}$ What we are concerned with is the time-dependent change of the turbidity, because it is this change which reflects how quickly particles of the system are aggregating. The turbidity measurement becomes possible only when the turbidity change of the system has some connection with the aggregation process. If the suspensions are not stable, then in the early stage of the aggregation only one process basically exists: two primary particles are combined to form a doublet. In this case, if $2 C_{1} \neq C_{2}$, there will be a corresponding change in extinction sections for the suspension resulting in the turbidity change of the system. The change rate of turbidity due to the aggregation of single particles can be written as: ${ }^{12,35}$

$$
\frac{\mathrm{d} \tau}{\mathrm{d} t}=C_{1} \frac{\mathrm{d} N_{1}}{\mathrm{~d} t}+C_{2} \frac{\mathrm{d} N_{2}}{\mathrm{~d} t}
$$

Combining eqn (1), (2) and (19), the CRC can be connected with the rate of turbidity change in the turbidity measurement by the following equation: ${ }^{35}$

$$
k_{11}=\frac{\left[\mathrm{d}\left(\tau / \tau_{0}\right) / \mathrm{d} t\right]_{0}}{\left[\left(C_{2} / 2 C_{1}\right)-1\right] N_{1}}
$$

where $\tau_{0}$ is the turbidity at the coagulation starting time $t=0$. Assuming the denominator in eqn (20) can be taken to be a constant, the coagulation rate will be proportional to the quantity of $R=\left[\mathrm{d}\left(\tau / \tau_{0}\right) / \mathrm{d} t\right]_{0}$, the relative rate of the turbidity change, which is the slope of the $\left(\tau / \tau_{0} \sim t\right)$ curve at $t=0$. Thus, $|R|$ is usually used to represent the relative coagulation rate. It has been shown ${ }^{12}$ that even for the same dispersions, the slope of the line of $\tau$ versus $t$ may be positive or negative, depending on the wavelength $\lambda$ used. Fig. 2 gives two typical curves of $\tau / \tau_{0}$ versus $t$ at two different wavelengths, for suspensions consisting of polystyrene (PS) spheres with radius $a=250 \mathrm{~nm}$. According to this type of plot, $R$ can be evaluated by linear fitting techniques for its linear portion near $t \approx 0$.

The plot in Fig. 3 shows the dependence of $R$ on $\lambda$ (as well as the dimensionless size parameter $\alpha=2 \pi a / \lambda)$ for suspensions consisting of PS particles $(a=250 \mathrm{~nm})$.

Usually, the size parameter is used to define particle size as measured by the operating wavelength. However, the characteristics of light scattering vary with the relative refractive index of the particle and the medium and, in general, the relative refractive index changes with the operating wavelength. ${ }^{36-38}$ Therefore, the properties of turbidity depend on both particle size and wavelength. Using the size parameter alone to describe the properties of turbidity may cause error. This is the reason why two parameters (particle size and wavelength) are needed, instead of the size parameter alone.

From Fig. 3, we can see that $R$ varies dramatically with $\lambda$ : not only is its magnitude very different at different $\lambda$, but its signs may also change from negative, through zero, to positive. When two primary particles are combined to form a doublet during the coagulation, there will be a corresponding change in their extinction sections. If $\left(2 C_{1}\right)<\left(C_{2}\right),(\mathrm{d} \tau / \mathrm{d} t)>0$ (namely $\left.R>0\right)$; if $\left(2 C_{1}\right)<\left(C_{2}\right), R<0$. That the case of $R=0$ corresponds to the extinction section of two singlets is, by coincidence, exactly equal to that of one doublet $\left(2 C_{1}=C_{2}\right)$. If the measurement is performed at the $\lambda$ with $R=0$, then $R$ will have no response to the actual coagulation process (the "blind point" for measurement). This means that in the zone of the $\lambda$ with $R \approx 0$, the change in the turbidity during coagulation completely loses its sensitivity to the

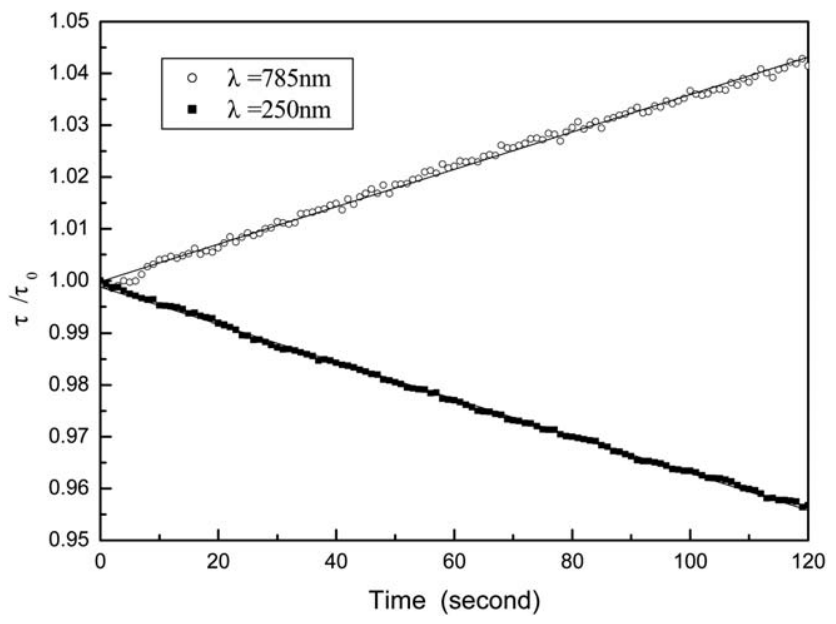

Fig. 2 Plot of the turbidity $\tau$ versus time $t$ for suspensions of particles with radius $a=250 \mathrm{~nm}$ at incident wavelengths. ${ }^{12}$ 


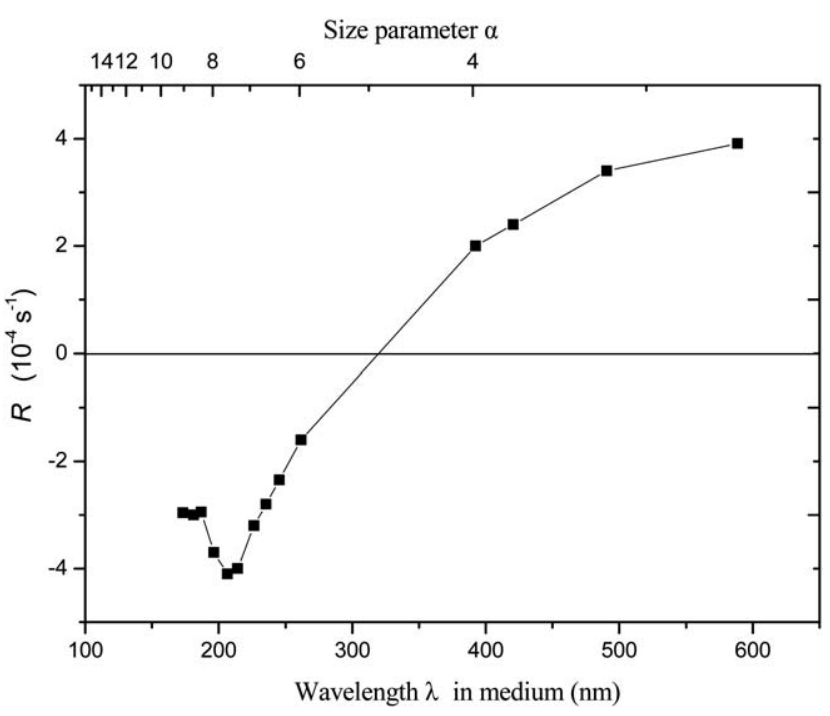

Fig. 3 The relative rate of the turbidity change at $t \rightarrow 0, R=\left(1 / \tau_{0}\right)(\mathrm{d} \tau /$ $\mathrm{d} t)_{0}$, versus wavelength $\lambda$ and the size parameter $\alpha .^{12}$

change in particle number $N$ of the suspensions. That is, there is a zero-sensitivity zone (or the blind zone) for the turbidity measurement around $\lambda=320 \mathrm{~nm}$, where $R$, the magnitude of the slope of $\tau-t$ curve at $t=0$, is too small to distinguish (the signal is covered by noise). ${ }^{12}$ Thus, to enhance the signal-to-noise ratio and reduce the uncertainties of the measurement, one should perform the turbidity measurement at a proper incident light wavelength $\lambda$, where the magnitude of $R$ is sufficiently large. ${ }^{12}$

As discussed above, at the initial stage of coagulation, we have $k \propto R$ for turbidity measurement. If its coefficient of proportionality has a constant value, $R$ has a connection with the stability ratio $W$, which is typically used to describe the degree of stability of a colloidal system. The stability ratio $W$ is commonly estimated by the ratio of the diffusion-controlled rapid coagulation rate $\left(k_{\text {rapid }}\right)$ to the coagulation rate $(k)$ of the system in consideration: ${ }^{22,39-41}$

$$
W=k_{\text {rapid }} / k=R_{\text {rapid }} / R
$$

The reciprocal of the stability ratio $1 / W$ is the so-called "sticking probability" or "collision efficiency". We can see that to obtain $W$, one needs not only a measurement of $R$ for the sample itself, but also must prepare an additional sample to acquire $R_{\text {rapid. }}$ For an unknown suspension, this is not always easy to do. On the other hand, the absolute rates do not need two measurements, and therefore are more desirable if they can be derived accurately from the turbidity measurement.

To determine the absolute coagulation rate constant (CRC) from turbidity measurements, one of the most difficult problems is how to achieve the dimensionless parameter $F=\left[\left(C_{2} / 2 C_{1}\right)-1\right]$, which is referred to as the optical factor, in the denominator of eqn (20). $R$ can be obtained from the turbidity measurement, but the optical factor has to be quantitatively calculated by means of light scattering theory.

The calculation of the optical factor is involved in the evaluation of extinction cross sections for single particle and doublet.

The Mie theory is valid for calculating the cross sections of spheres without size limit. ${ }^{4344}$ It therefore has no difficulty accurately calculating $C_{1}$. The Mie theory, however, cannot evaluate $C_{2}$ correctly, because it is associated with the extinction cross section (or scattering cross section, for a nonabsorbing particle) of a doublet composed of two spheres. One way to deal with this problem is to adopt the coalescing assumption for a doublet, in which the aggregated doublet is considered to coalesce into a spherical particle with the same volume of the two separated particles. Apparently, the coalescence does not actually take place (probably, liquid droplet is the only exception), so the error associated with this coalescence assumption is unavoidable in the calculation of the optical factor. We will refer to this approximation method as Mie(coalescence), below.

Similarly to as discussed in Section 2.1, the RGD approximation can also be used to evaluate the extinction cross sections of a single particle and real doublet for small particles in the turbidity measurement. The calculation of the optical factor with RGD approximation will be referred to as RGD(real).

The RGD method can also be used to calculate the optical factor with the coalescence assumption, and this variation of the RGD method will be referred to as RGD(coalescence). Using the results of Mie(coalescence) and RGD(coalescence), Lichtenbelt et $a l .{ }^{35}$ have tried to improve the calculation of the optical factor by correcting the RGD(real) results. The corrected optical factor is calculated using the formula: ${ }^{35}$

$$
\frac{C_{2}}{2 C_{1}}-1=\frac{C_{2}{ }^{\prime}(\mathrm{Mie}) C_{2}(\mathrm{RGD})}{2 C_{1}(\mathrm{Mie}) C_{2}{ }^{\prime}(\mathrm{RGD})}-1
$$

where $C_{2}{ }^{\prime}$ (Mie), $C_{2}$ (RGD) and $C_{2}{ }^{\prime}$ (RGD) are calculated from Mie(coalescence), RGD(real) and RGD(coalescence), respectively. $C_{1}(\mathrm{Mie})$ is calculated from Mie theory. We will refer to this method as RGD(corrected).

Thus, the measurement of CRC by using the turbidity measurement with the extinction cross section of doublets, calculated based on the above theories, can yield a reasonable approximation only for small particles.

It has been pointed out ${ }^{12}$ that there are some inherent problems when small particle suspension is used in the turbidity measurement. For small particles, a high-number concentration of particles is usually required in order to enhance the signal-tonoise ratio in the measurement. This is because scattering intensity decreases towards smaller particles with the sixth power of radius, and suspensions of small particles have very low turbidity. In this case, a dispersion of smaller particles at lownumber concentration become an almost transparent medium, and therefore the change in its turbidity becomes very difficult to detect accurately. The turbidity of smaller particles can be enhanced by increasing the number-concentration of particles. However, a high-number concentration of particles will make the coagulation occur too quickly, thus causing the linear portion of the turbidity-time curve to become very short before it curves. In this case, the measurement usually needs to be done within just several seconds. ${ }^{39}$ This causes large errors, associated with the data fluctuations and the insufficient data collection time. In contrast to small particles, when large particles with a lownumber concentration are used, the linear portion of the curve of $\tau / \tau_{0}-t$ can be quite long, as shown for particles of $a=250 \mathrm{~nm}$ in Fig. 1, thus allowing the measurement of $R$ to be more accurate. 
To solve the problem with a quickly-curved line of $\left(\tau / \tau_{0} \sim t\right)$ when a high number-concentration of particles is used, Puertas et $a l .{ }^{45}$ applied the RGD approximation to calculating the total light scattering cross section of the aggregate, taking into account the contributions from pairs of particles with zero, one, and two particles between them, and developed a technique for getting the $\mathrm{CRC}$ from the turbidity measurement by fitting the curve of turbidity change for a longer time.

An additional disadvantage of using small particles is that, if the methodology is only for small particles and small $\alpha$, one will greatly restrict the range of $\lambda$ which can be selected. As mentioned above, $R$ varies with $\lambda$, so to reduce experimental errors one should choose a $\lambda$ at which $R$ is large. ${ }^{12}$

Using the T-matrix technique, the extinction cross sections of single particles and doublets, and thus the optical factor $F$, can be calculated without additional assumptions. In the T-matrix method, both incident and scattered electric fields are expanded in a series of vector spherical wave functions as follows: ${ }^{31}$

$$
\begin{array}{r}
\mathbf{E}^{i n c}(\mathbf{r})=\sum_{n=1}^{\infty} \sum_{m=-n}^{n}\left[a_{m n} \operatorname{Rg} \mathbf{M}_{m n}(k r)+b_{m n} \operatorname{Rg} \mathbf{N}_{m n}(k r)\right] \\
\mathbf{E}^{s c a}(\mathbf{r})=\sum_{n=1}^{\infty} \sum_{m=-n}^{n}\left[p_{m n} \mathbf{M}_{m n}(k r)+q_{m n} \mathbf{N}_{m n}(k r)\right]
\end{array}
$$

where $k=2 \pi / \lambda$. Due to the linearity of Maxwell's equations, the scattered field coefficients $\mathbf{p}=\left[p_{m n}, q_{m n}\right]$ are related to the incident field coefficients $\mathbf{a}=\left[a_{m n}, b_{m n}\right]$ by means of the so-called transmission matrix (or T matrix):

$$
\begin{aligned}
& p_{m n}=\sum_{n^{\prime}=1}^{\infty} \sum_{m^{\prime}=-n^{\prime}}^{n^{\prime}}\left[T_{m n m^{\prime} n^{\prime}}^{11} a_{m^{\prime} n^{\prime}}+T_{m n m^{\prime} n^{\prime}}^{12} b_{m^{\prime} n^{\prime}}\right] \\
& q_{m n}=\sum_{n^{\prime}=1}^{\infty} \sum_{m^{\prime}=-n^{\prime}}^{n^{\prime}}\left[T_{m n m^{\prime} n^{\prime}}^{21} a_{m^{\prime} n^{\prime}}+T_{m n m^{\prime} n^{\prime}}^{22} b_{m^{\prime} n^{\prime}}\right]
\end{aligned}
$$

In compact matrix notation, eqn (25) and (26) can be rewritten as:

$$
\left[\begin{array}{l}
\mathbf{p} \\
\mathbf{q}
\end{array}\right]=\mathbf{T}\left[\begin{array}{l}
\mathbf{a} \\
\mathbf{b}
\end{array}\right]=\left[\begin{array}{ll}
\mathbf{T}^{11} & \mathbf{T}^{12} \\
\mathbf{T}^{21} & \mathbf{T}^{22}
\end{array}\right]\left[\begin{array}{l}
\mathbf{a} \\
\mathbf{b}
\end{array}\right]
$$

which means that the column vector of the expansion coefficients of the scattered field is obtained by multiplying the $\mathrm{T}$ matrix and the column vector of the expansion coefficients of the incident field. After the computation of the $\mathrm{T}$ matrix for single particles and doublets, their extinction cross sections can be calculated by:

$$
\left\langle C_{\text {ext }}\right\rangle=-\frac{2 \pi}{k^{2}} \operatorname{Re} \sum_{n=1}^{\infty} \sum_{m=-n}^{n}\left[T_{m n m n}^{11}+T_{m n m n}^{22}\right]
$$

Then, one can get the optical factor $F$ from the calculated results of $C_{2}$ and $C_{1}$ by eqn (28). More comprehensive discussion of the technical details and data calculations of the optical factor for different particle sizes and wavelengths is presented in ref. 46 . The T-matrix method has been successfully applied to calculating the extinction cross section of a real doublet for homocoagulation, ${ }^{12,46}$ as well as heterocoagulation. ${ }^{47}$ More recently, this approach has been extended to the measurement of turbulent coagulation. $^{48}$
To assess the quality of various theoretical treatments (calculation of the optical factor $F$ ) dealing with the turbidity measurement for determining the rate constants, a useful criterion was proposed. ${ }^{12}$ This criterion is based on a reasonable prediction that optical factor $F$ should ensure that eqn (20) yields an identical $\mathrm{CRC}$, no matter what wavelength is used in the turbidity measurement.

Comparisons for the absolute CRCs determined by eqn (20), with the optical factor derived from the T-matrix method and other theoretical approaches, are presented in Fig. 4, 5 and $6 .^{12}$ All points of the rate constants in each figure are based on the same original experimental data - namely, the slopes of the linear portion of the $\left(\tau / \tau_{0} \sim t\right)$ curves at different incident light wavelengths. In all of these Figures, two different scales are used for

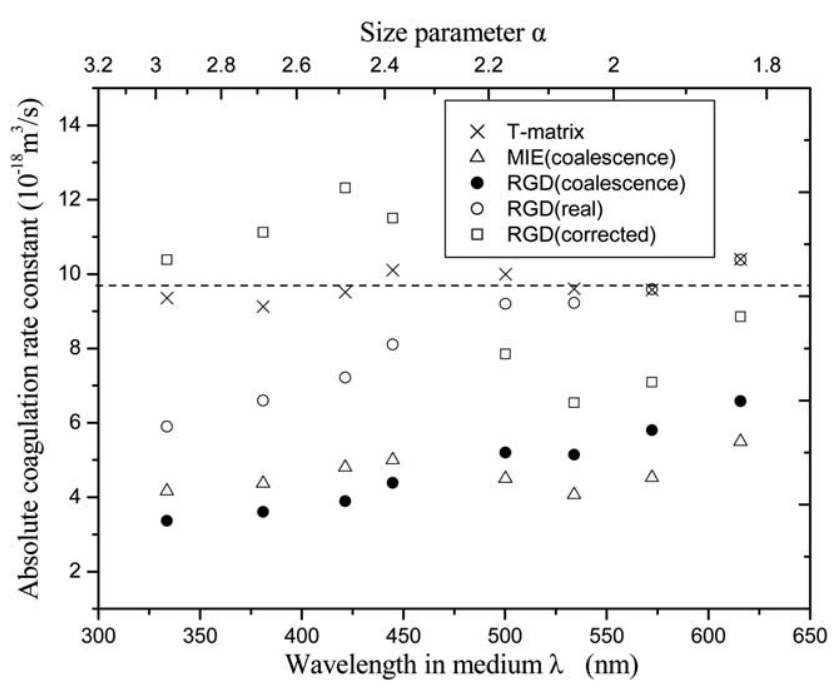

Fig. 4 Absolute coagulation rate constants (CRC) derived from different theoretical treatments for suspensions of particles with radii $a=$ $170 \mathrm{~nm}^{12}$

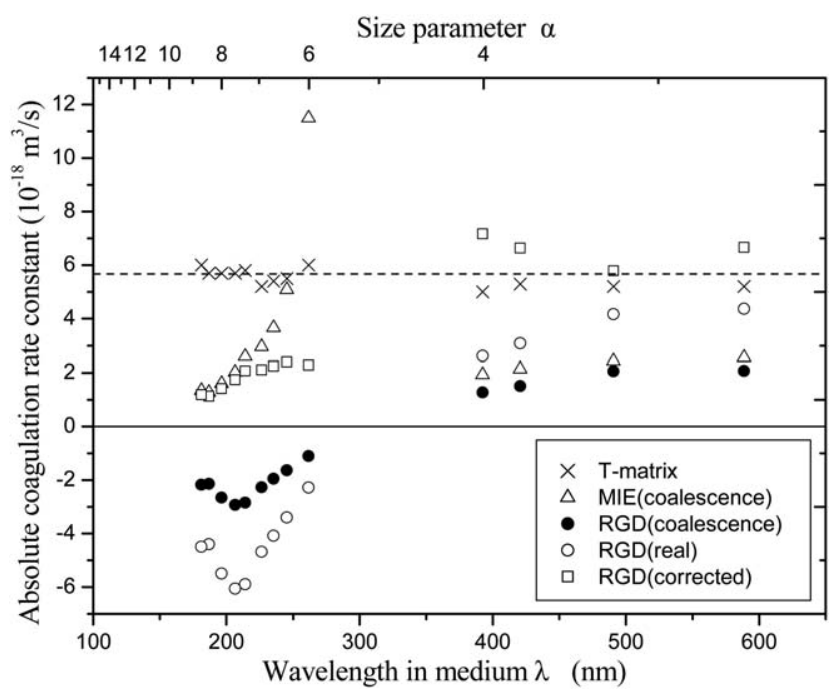

Fig. 5 Absolute coagulation rate constants (CRC) derived from different theoretical treatments for suspensions of particles with $a=250$ $\mathrm{nm}{ }^{12}$ 


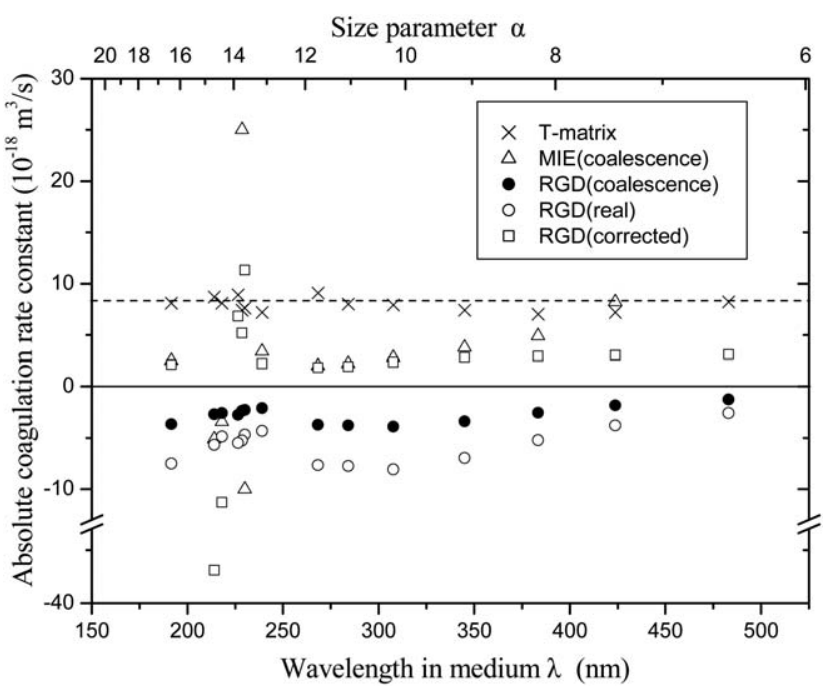

Fig. 6 Absolute coagulation rate constants (CRC) derived from different theoretical treatments for suspensions of particles with $a=500$ nm. ${ }^{12}$

each abscissa, and are marked by both the wavelength $\lambda$ and the size parameter $\alpha$. The particle sizes of $a=170 \mathrm{~nm}, 250 \mathrm{~nm}$ and $500 \mathrm{~nm}$ are used, respectively, in Fig. 4, Fig. 5 and Fig. $6 .{ }^{12}$ These plots show that except for the T-matrix method, none of the previous theories can give an unaltered quantity for the whole range of the wavelength $\lambda$, or the size parameter $\alpha$, used in the experiments. There are some theories which, in many cases, even yield negative values for the rate constants; these values are apparently meaningless.

Fig. 4 (Suspensions of particles with radii $a=170 \mathrm{~nm}$ ) shows that the data from the RGD(real) and T-matrix are actually overlapping when $\alpha \leq 2$, meaning that, for small particles and small $\alpha$, the T-matrix treatment coincides with RGD(real) method. This fact indirectly proves that use of the T-matrix solution is correct, because it is known that the RGD(real) is a good approximation when treating doublets for small $\alpha$ and small particles. In addition, for these-sized particles, all the data of the rate constants from the four previous theories are basically within the same order of magnitude when $\alpha<3$, although still showing significant deviations from a constant value.

Fig. 5 (Suspensions for particles of $a=250 \mathrm{~nm}$ ) shows that among the four previously discussed theories, the RGD(corrected) has the best performance and the RGD(real) comes in second, when $\alpha<4$. There is no data available in the zone $(4<\alpha<6)$ because of the zero sensitivity zone, as previously mentioned. When $\alpha>6$, RGD(coalescence) and RGD(real) yield negative values for the rate constants, making those results meaningless.

Fig. 6 (Suspensions for particles of $a=500 \mathrm{~nm}$ ) shows that all previous theories failed to give reasonable data for the rate constant for such-sized suspensions when $\alpha>6$. Therefore, the T-matrix solution is the only surviving method with an excellent performance throughout the whole $\alpha$ range.

\subsection{Other related work}

3.2.1 A simple scheme for obtaining the turbidity change rate vs. wavelength. As discussed above, the knowledge of turbidity

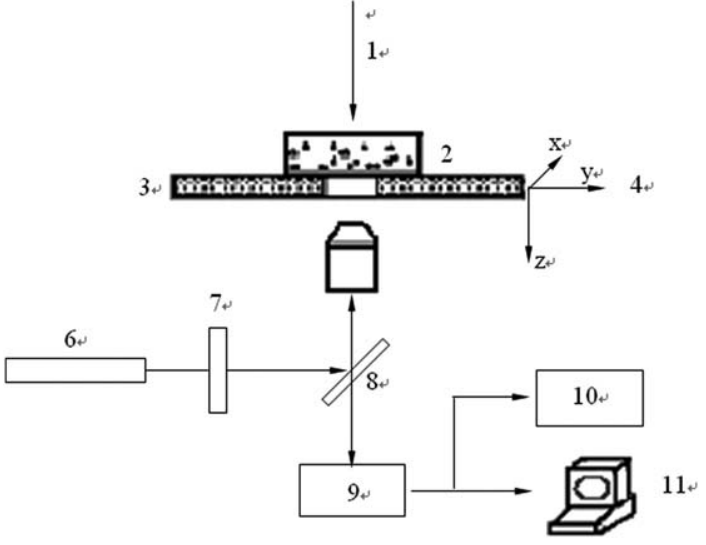

Fig. 7 Experimental setup: $1=$ illumination light; $2=$ sample cell; $3=$ sample stage; $4=3 \mathrm{D}$ motion; $5=$ Objective; $6=$ light source of optical tweezers; $7=$ electronic shutter; $8=$ dichromatic mirror; $9=\mathrm{CCD}$ camera; $10=$ video recorder; $11=$ computer ${ }^{42}$

derivatives with respect to time $(\mathrm{d} \tau / \mathrm{d} t)$ over a range of operating wavelengths is very helpful for more accurately and effectively evaluating the coagulation rate by using turbidity measurement. A rough-and-ready curve of $(\mathrm{d} \tau / \mathrm{d} t) v s$. $\lambda$, like Fig. 3, can help one to easily select an appropriate wavelength for performing the turbidity measurement, not only apart from the blind point, but with enough sensitivity to the coagulation. A simple scheme for producing such a curve by means of the spectrophotometer has been proposed. ${ }^{47}$

A brief summary of this scheme ${ }^{47}$ is that two turbidity scans over a range of the wavelength are performed during a short time-interval by means of the spectrophotometer, and then the time-differences of turbidity $(\Delta \tau / \Delta t)$ are obtained as approximations to their time-derivatives $(\mathrm{d} \tau / \mathrm{d} t)$.

3.2.2 Heterocoagulation rate constant of dispersions containing particles of different sizes. Heterocoagulation exhibits more complicated behavior than homocoagulation, and is therefore more difficult to study through modeling. Therefore, as a relatively simple example, heterocoagulation of two differently-sized colloidal particles has received significant attention.9,10,49 The main points for the turbidity measurement of the monocoagulation rate summarized above are also applicable to the case of heterocoagulation.

Compared with homocoagulation, two points should be addressed for studying heterocoagulation by turbidity measurement: ${ }^{47}$ (A) For heterocoagulation, the blind point shifts with the component fraction, while for homocoagulation the blind point is fixed. (B) Since turbidity increases radically with particle size, larger particles may significantly dominate the turbidity behavior of the binary dispersion, resulting in large measurement error. This tendency becomes stronger when constituent particles of the dispersion are smaller. Properly increasing the component fraction of small particles is helpful for diminishing this problem.

3.2.3 Uncertainties caused by the forward scattering in turbidity measurement of the coagulation rate. As discussed above, if possible, it would be preferable to reduce the error in turbidity measurement by choosing larger particles. ${ }^{12}$ However, 
this does not necessarily mean 'the larger the better' for particle sizes used in turbidity measurement. This is because the forward scattering light (FSL) received by the detector can cause uncertainties in turbidity measurement of coagulation rate of a colloidal dispersion, and this effect becomes more significant for large particles. In general, acceptance angles for actual turbidity measurement instruments or commercial spectrophotometers are in the range of a few degrees. ${ }^{7,50}$ In this case, a portion of FSL may fall with the transmitted light onto the light detector, thus causing an error in the turbidity measurements. ${ }^{51}$ When worrying about FSL effects for larger particles, one may lack confidence in results when using large particles in the turbidity measurements, unless one possesses the necessary knowledge of how to reduce and control for the FSL effect.

A direct way to reduce the error caused by FSL is by making the acceptance angle as small as possible in the experimental setup for the turbidity measurement. Special efforts have been made $^{52}$ toward this goal; for best results, the acceptance angle can be reduced to close to 0 .

On the other hand, carefully selecting particle size and measuring wavelength can also significantly reduce the error caused by FSL. The theoretical formulation and relevant numerical implementation for predicting the contribution of FSL under various parametric conditions in the turbidity measurement have been presented. ${ }^{51}$ This investigation provides a useful guideline for properly choosing particle size, and measuring wavelength (to minimize the effect of FSL). It also provides a formula for correcting errors caused by the effect of FSL when geometrical parameters related to the detector, such as the acceptance angle, are available. As an example, for an acceptance angle of $3^{\circ}$, in order to ensure that the relative error in measurement caused by FSL is smaller than 5\%, we need to select a particle size and measuring wavelength to satisfy the condition: $\alpha<4$ or $8<\alpha<11$. For particles with diameters around $1.5 \mu \mathrm{m}$, this condition means that the selectable wavelength $\lambda$ should be in the range: $0.57 \mu \mathrm{m}<\lambda<0.78 \mu \mathrm{m}$. More detailed information is described in ref. 51 .

3.2.4 Progress on determining refractive indices of medium and dispersed particles. The refractive indices of materials are very fundamental parameters for light scattering measurement, including for turbidity. To accurately calculate the extinction cross sections or optical factor, the refractive indices $n_{2}$ and $n_{1}$ of the particle and the medium have to be predetermined with sufficient accuracy. As previously indicated, selecting proper operating wavelengths to perform the rate-constant measurements is very important. In general, the refractive indices $n_{2}$ and $n_{1}$ vary with the operating wavelength. ${ }^{36-38}$ Recent studies ${ }^{12,46}$ have shown that even a tiny error in the refractive indices may result in significant errors in calculations of the optical factor in the turbidity measurement; this may lead to large errors in measuring CRC. Therefore, how one determines the values of $n_{2}$ and $n_{1}$ at desirable wavelengths poses a challenging problem.

For some commonly used materials in colloids, such as polystyrene and water, the refractive indices are available at some discrete wavelengths. ${ }^{36-38}$ When the experiment is performed at the wavelengths where the values of $n_{2}$ and $n_{1}$ are unknown, one has to resort to some empirical formulas or fitting formulas. ${ }^{36,37,46}$ Data obtained via such methods for $n_{2}$ and $n_{1}$ may have significant uncertainty, particularly if the fitting data points are sparse or far away from the operating wavelength. Furthermore, the introduction of ions or polymers into the medium may change the refractive index of the medium, such that the data for the pure medium may not suit the environment in the actual experiment.

A new method for measuring refractive indices in situ at the desired wavelength $\lambda$ has been developed ${ }^{53}$ In this method, the refractive indices of both the dispersed particles and the medium can be inversely deduced, based on Mie theory, from the extinction cross sections of monodispersed particles via the turbidity measurement.

3.2.5 Influence of multiple scattering effect in light-scatteringbased applications. Many applications of the light scattering techniques are based on the single-scattering approximation, in which the light scattering properties of single particles are expected to represent the behavior of the entire ensemble. Therefore, the volume fraction should be carefully selected to ensure that the multiple scattering effect becomes negligible in light-scattering experiments.

The multiple scattering effects in light scattering experiments were investigated using $\mathrm{T}$-matrix method, ${ }^{54,55}$ and thus the allowed volume fractions for given accuracy requirements of single-scattering approximation can be determined.

\section{Additional comments}

In the last section, we summarize some major results from recent research on turbidity measurement. Most of these results are also instructive for other light scattering methods discussed in Section 2 , because of their similarity. Compared with regular forms used in static and dynamic light scattering, the expressions for the CRC $\left(k_{11}\right)$, such as eqn (6) and (15), have been adjusted in Section 2 to make them more comparable with that for turbidity in Section 3.

In turbidity methods, we have $k \propto\left[\mathrm{d}\left(\tau / \tau_{0}\right) / \mathrm{d} t\right]_{0}$. Similarly, in static and dynamic light scattering, we have $k \propto[\mathrm{d} I(q, t) / \mathrm{d} t]_{0} / I$ $(q, 0)$ and $k \propto\left[\mathrm{d}\left(r_{\mathrm{h}}(t) / r_{\mathrm{h}}(0)\right) / \mathrm{d} t\right]_{0}$, respectively, according to eqn (6) and eqn (15). By estimating the rate at which $I(q)$ or $r_{\mathrm{h}}$ change with time in static or dynamic light scattering at the very beginning of the coagulation, the relative coagulation rate can also be obtained. The stability ratio $W$ then becomes available from eqn (21).

As mentioned before, the measurement sensitivity of the turbidity method depends on the operating wavelength (when the particle size is fixed), and its zero sensitivity is corresponding to the blind point where $C_{2}=2 C_{1}$. For the same reason, in static light scattering, the measurement sensitivity should depend on scattering vector $q$ or the scattering angle (for fixed wavelength), and it is expected that there would also be a blind point (zero sensitivity) at a special scattering angle where $I_{2}(q)=2 I_{1}(q)$. The scattering angle should be appropriately selected to ensure that the light-scattering-change rate is sufficiently sensitive to the coagulation process.

It is probably worth emphasizing here that all the methods for determining the absolute CRCs discussed above are valid only for suspensions of spherical particles. This is because the value of $C_{2}$ (for turbidity methods) or $I_{2}(q)$ (for scattering methods) can 
be mathematically calculated from theories only for spherical particles. Although we do not need the value of $I_{2}(q)$ for simultaneous static and dynamic light scattering, the hydrodynamic factor $\left(1-r_{\mathrm{h}, 1} / r_{\mathrm{h}, 2}\right)$ in eqn (17) can still only be achieved for spherical particles.

\section{A microscopic approach to evaluate the colloidal stability}

The methods of determining coagulation rates, such as light scattering and turbidity, are based on information on the longterm, accumulated effects of the motions and interactions of huge numbers of particles during coagulation; thus, they can be called "macroscopic approaches". ${ }^{42}$ In contrast, the "microscopic approach" is meant to assess the colloidal stability at individual particle levels, by means of directly observing artificially induced collision.

The stability ratio $W$ of eqn (21) is physically equal to: the ratio of total number of particle collisions $(n)$ to number of collisions leading to permanent doublets $\left(n_{\mathrm{c}}\right)^{: 40,41}$

$$
W=n / n_{\mathrm{c}}
$$

As previously mentioned, the reciprocal of $W$ is the "sticking probability", $p=n_{\mathrm{c}} / n{ }^{56-58}$ For slow coagulation or reactionlimited cluster aggregation (RLCA), the energy barrier prevents every encounter from becoming effective aggregation. Only a fraction of collisions, $1 / W$, is successful. The difficulty of using eqn (29) is that Brownian motions make particle collisions take place at unpredictable locations; therefore, one has no way to observe particle collisions and their outcomes in order to get a true $n_{\mathrm{c}}$. To solve this problem, optical tweezers (or optical traps $)^{59,60}$ have been used ${ }^{42}$ to catch two particles, and bring them together for a collision in an area observable by a microscope (see Fig. 7). By observing this "artificially induced collision," it is possible to check what occurs when they are released from the trap: namely, whether they stick together or continue to separate after their collisions. After sampling $n$ pairs of particles for such artificial collisions, a "sticking probability" can be estimated by the ratio of the number of sticking pairs $n_{\mathrm{c}}$ to non-sticking pairs $n$.

When two particles are caught in the optical trap at time $t=$ 0 and released at the time $t=t_{\text {dur }}$, the total (or accumulated) sticking probability $P\left(t_{\mathrm{dur}}\right)$ for the particle pair to stick together can be deduced to have the following expression ${ }^{42}$ :

$$
P=1-\mathrm{e}^{-p f t}
$$

where $p$ is the sticking probability after a single collision of a particle pair in the optical trap, and $f$ is the collision frequency in the trap.

Fig. 8 shows a typical curve of the sticking probability $P$ vs. $t$, obtained from experiment. ${ }^{42}$ This curve, showing exponential growth of $P$ with $t_{\text {dur }}$, is basically consistent with prediction of eqn (30). The major difference is that there is a great jump at the beginning of the induced collision, instead of starting from zero at $t=0$, as indicated by eqn (30).

A possible explanation for this difference is that, at the beginning of trapping, the trapping speed brings the particle pair

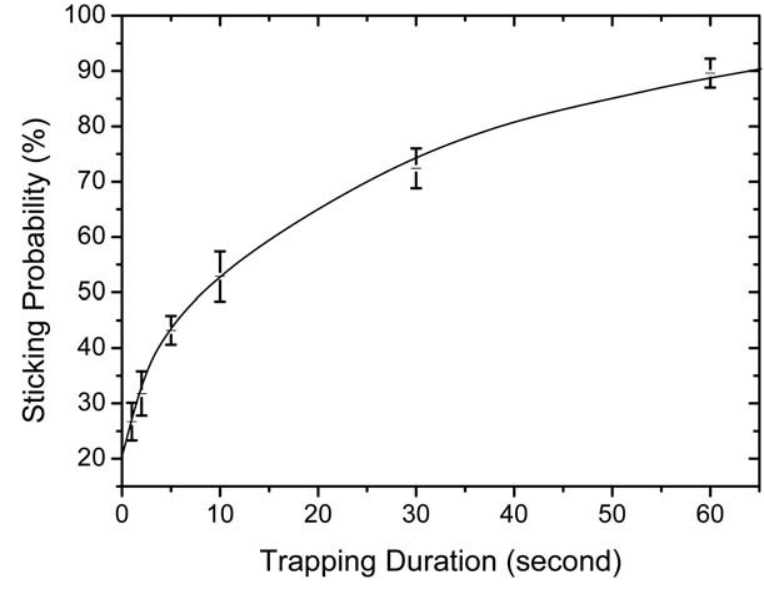

Fig. 8 The dependency of the total sticking probability $P$ on the trapping duration $t_{\text {dur. }}{ }^{42}$

into the "compact status" with higher collision frequency, and then quickly falls into the "relaxed status" with lower collision frequency. ${ }^{61,62}$ This change in collision frequency causes an abnormally increasing rate of total sticking probability $P$ to appear at the beginning, and then diminishes to a smaller, normally-increasing rate corresponding to the relaxed status. The approaches for achieving the sticking probability $f$ (or the stability ratio $W$ ) from the experiments of artificially induced collisions have been proposed, and their results for the stability ratios $W$ are consistent with those from turbidity measurements. ${ }^{42,63}$

One of the superior features of the microscopic approach as compared to the turbidity method is that the former needs only to perform measurements on the suspension sample itself; the latter needs not only the sample itself for $k$ evaluation, but one must also prepare an additional sample to acquire $k_{\text {rapid. }}$. For an unknown suspension, this is not always easy to do. For instance, the methods using eqn (21) cannot, in general, be applied to sterically stabilized systems. ${ }^{42}$ An additional advantage of this procedure is that only a small amount of dispersion solution is needed for sampling tests (about $0.1 \mathrm{~mL}$ ). The disadvantage of this approach is that its procedure is quite time-consuming.

The reason for existence of two statuses of the artificially induced collisions - "compact" and "relaxed" - in an optical trap is explained by a Brownian dynamics simulation on the collisionsticking dynamics of two colloidal particles in an optical trap. ${ }^{64}$ In this simulation, various contributing factors - including the DLVO (Derjaguin-Landau-Verwey-Oberbeek) interaction of particles, hydrodynamic interactions, optical trapping forces on the two particles, and the Brownian motion - were all taken into account. The simulation results have reproduced the relevant features of the $\ln (1-P) \sim t_{\text {dur }}$ curve found in the experiments.

\section{Summary}

This review summarizes recent advances in coagulation rate measurements of colloidal dispersions, with emphasis on turbidity method.

For the light-scattering methods, the primary research efforts have revolved around solving the problem of the form factor. Several theoretical methods including DDA, MA and the 
T-matrix method have been applied to calculating the form factor, while the approach of SSDLS artfully bypassed the calculation of this quantity. Apparently, the T-matrix method is more promising.

The recent studies on the turbidity measurement contribute to deepening an understanding of the mechanisms: how major factors influence measurement sensitivity and accuracy. Theoretical analysis and experiments clearly confirmed that the measuring sensitivity significantly depends on particle size and the wavelength used. Particularly, there exists a "zero sensitivity" blind point for measurement at a specific wavelength. Therefore, measurement is suggested to be performed at a wavelength away from the blind point, with sufficient sensitivity to detect the coagulation rate. For evaluation of the extinction cross section of the doublet, the T-matrix method provides an accurate and robust solution, and therefore removes the restriction on particle size and increases measurement accuracy. The studies also advocate that to achieve a more accurate absolute CRC by enhancing the signal-to-noise ratio and reducing the uncertainties of the measurement, a moderate turbidity would be appropriate for the turbidity measurement; this condition can be easily satisfied by using larger particles.

Heterocoagulation exhibits more complicated behavior because the blind point shifts with the component fraction, and larger particles may significantly dominate the turbidity behavior of the binary dispersion, resulting in large measurement error.

The turbidity derivative with respect to time $(\mathrm{d} \tau / \mathrm{d} t)$ corresponds to the measurement sensitivity of the coagulation rate, and a high sensitivity is desirable for more accurate measurement. The proposed scheme to produce a rough-and-ready curve of $(\mathrm{d} \tau / \mathrm{d} t) v s . \lambda$ is helpful for selecting an appropriate wavelength for the turbidity measurement.

The errors caused by FSL in turbidity measurements of coagulation rate become more significant for larger particles. The investigation on the effect of FSL provides a useful guideline for properly choosing particle size and measuring wavelength, as well as a formula for correcting errors caused by the effect of FSL.

The refractive indices are important data for the light scattering measurement. The inverse method presents a new approach to determine refractive indices at the desired wavelength $\lambda$ through the turbidity measurement.

The similarity and comparison between the turbidity method and other light scattering methods is particularly emphasized in this article. In this way, two types of approaches can exchange and share each other's achievements in their research. For instance, the concept of a blind point shown in the turbidity measurement is also expected in the light scattering method, although it has not yet been previously reported in the literature.

In contrast with traditional measurement methods, in the last section, the "microscopic approach" to assess the colloidal stability is introduced. This approach checks the outcomes from artificially induced collisions at individual particle levels, by means of directly observing artificially induced collisions with the aid of optical tweezers.

\section{Acknowledgements}

We thank Mr Michael R. Honig for his help in editing this manuscript. This work is supported by Grants 10972217,
10932012 and 11032011 from the National Natural Science Foundation of China.

\section{References}

1 R. D. Vold and M. J. Vold, Colloid and Interface Chemistry, AddisonWesley, London, 1983.

2 A. Lips, C. Smart and E. Willis, Trans. Faraday Soc., 1971, 67, 2979 2988.

3 R. Folkersma, A. J. G. van Diemen and H. N. Stein, J. Colloid Interface Sci., 1998, 206, 482-493.

4 D. L. Swift and S. K. Friedlander, J. Colloid Sci., 1964, 19, 621-647.

5 M. Elimelech, J. Gregory, X. Jia and R. A. Williams, Particle Deposition and Aggregation, Butterworth-Heinemann, Oxford, England, 1995.

6 H. Reerink and J. T. G. Overbeek, Discuss. Faraday Soc., 1954, 18, $74-84$.

7 J. Gregory, Adv. Colloid Interface Sci., 2009, 147-148, 109-123.

$8 \mathrm{H}$. Holthoff, S. U. Egelhaaf, M. Borkovec, P. Schurtenberger and H. Sticher, Langmuir, 1996, 12, 5541-5549.

9 W. L. Yu, E. Matijevic and M. Borkovec, Langmuir, 2002, 18, $7853-$ 7860 .

10 W. Lin, M. Kobayashi, M. Skarba, C. Mu, P. Galletto and M. Borkovec, Langmuir, 2006, 22, 1038-1047.

11 M. von Smoluchowski, Z. Phys. Chem., 1917, 92, 129-168.

12 Z. W. Sun, J. Liu and S. H. Xu, Langmuir, 2006, 22, 4946-4951.

13 M. Kerker, The Light Scattering of Light and Other Electromagnetic Radiation, Academic Press, New York, 1969.

14 H. Holthoff, M. Borkovec and P. Schurtenberger, Phys. Rev. E: Stat. Phys., Plasmas, Fluids, Relat. Interdiscip. Top., 1997, 56, 69456953.

15 J. W. T. Lichtenbelt, C. Pathmamanoharan and P. H. Wiersema, J. Colloid Interface Sci., 1974, 49, 281-285.

16 H. Matsuoka, H. Murai and N. Ise, Phys. Rev. B, 1988, 37, 13681375.

17 P. Galletto, W. Lin, M. I. Mishchenko and M. Borkovec, J. Chem. Phys., 2005, 123, 064709.

18 P. Galletto, W. Lin and M. Borkovec, Phys. Chem. Chem. Phys., 2005, 7, 1464-1471.

19 J. H. van Zanten and M. Elimelech, J. Colloid Interface Sci., 1992, 154, $1-7$.

20 W. D. Young and D. C. Prieve, Langmuir, 1991, 7, 2887-2892.

21 G. R. Zeichner and W. R. Schowalter, J. Colloid Interface Sci., 1979, 71, 237-253.

22 G. Gillies, W. Lin and M. Borkovec, J. Phys. Chem. B, 2007, 111, $8626-8633$

23 J. W. Virden and J. C. Berg, J. Colloid Interface Sci., 1992, 149, 528535.

24 J. Happel and H. Brenner, Low Reynold's Number Hydrodynamics, Kluwer, Boston, 1973.

25 H. Holthoff, A. Schmitt, A. Fernandez-Barbero, M. Borkovec, M. A. Cabrerizo- Vilchez, P. Schurtenberger and R. HidalgoAlvarez, J. Colloid Interface Sci., 1997, 192, 463-470.

26 W. L. Yu, F. Bouyer and M. Borkovec, J. Colloid Interface Sci., 2001, 241, 392-399.

27 F. Bouyer, A. Robben, W. L. Yu and M. Borkovec, Langmuir, 2001, 17, 5225-5231.

28 M. Berka and J. A. Rice, Langmuir, 2004, 20, 6152-6157.

29 B. T. Draine and P. J. Flatau, J. Opt. Soc. Am. A, 1994, 11, 14911499.

30 D. W. Mackowski, Proc. R. Soc. London, Ser. A, 1991, 433, 599-614.

31 M. I. Mishchenko, L. D. Travis and A. A. Lacis, Scattering, Absorption, and Emission of Light by Small Particles, Cambridge University Press: Cambridge, U.K., 2002.

32 A. Quirantes and A. Delgado, J. Quant. Spectrosc. Radiat. Transfer, 2003, 78, 179-186.

33 M. I. Mishchenko, J. Opt. Soc. Am. A, 1991, 8, 871-882.

34 D. W. Mackowski, J. Opt. Soc. Am. A, 1994, 11, 2851-2861.

35 J. W. T. Lichtenbelt, H. J. M. C. Ras and P. H. Wiersema, J. Colloid Interface Sci., 1974, 46, 522-527.

36 X. Quan and E. S. Fry, Appl. Opt., 1995, 34, 3477-3480.

37 X. Ma, J. Q. Lu, R. S. Brock, K. M. Jacobs, P. Yang and X. H. Hu, Phys. Med. Biol., 2003, 48, 4165-4172.

38 I. D. Nikolov and C. D. Ivanov, Appl. Opt., 2000, 39, 2067-2070. 
39 H. Sonntag and K. Strenge, Coagulation Kinetics and Structure Formation, VEB Deutscher Verlag der Wissenschaften, Berlin, 1987.

40 R. J. Hunter, Zeta Potential in Colloid Science: principles and applications, Academic Press, London, 1981.

41 N. Fuchs, Z. Phys., 1934, 89, 736-743.

42 Z. W. Sun, S. H. Xu, G. L. Dai, Y. M. Li, L. R. Lou, Q. S. Liu and R. Z. Zhu, J. Chem. Phys., 2003, 119, 2399-2405.

43 A. J. Cox, A. J. Deweerd and J. Linden, Am. J. Phys., 2002, 70, 620625.

44 H. C. van de Hulst, Light Scattering by Small Particles, Wiley, New York, 1957.

45 A. M. Puertas and F. J. de las Nieves, J. Phys.: Condens. Matter, 1997, 9, 3313-3320.

46 S. H. Xu, J. Liu and Z. W. Sun, J. Colloid Interface Sci., 2006, 304, $107-114$.

47 J. Liu, S. H. Xu and Z. W. Sun, Langmuir, 2007, 23, 11451-11457.

48 M. Kobayashi and D. Ishibashi, Colloid Polym. Sci., 2011, 289, 831836.

49 J. M. Lopez-Lopez, A. Schmitt, A. Moncho-Jorda and R. HidalgoAlvarez, Soft Matter, 2006, 2, 1025-1042.

50 L. Wind and W. W. Szymanski, Meas. Sci. Technol., 2002, 13, 270275.

51 S. H. Xu and Z. W. Sun, Langmuir, 2010, 26, 6908-6918.
52 F. D. Bryant, B. A. Seiber and P. Latimer, Arch. Biochem. Biophys., 1969, 135, 97-108.

53 S. H. Xu, J. Liu, Z. W. Sun and P. Zhang, J. Colloid Interface Sci., 2008, 326, 110-116.

54 A. Quirantes, F. Arroyo and J. Quirantes-Ros, J. Colloid Interface Sci., 2001, 240, 78-82.

55 S. H. Xu and Z. W. Sun, Chin. Phys. Lett., 2007, 24, $1763-$ 1766.

56 M. Y. Han, H. K. Lee, D. F. Lawler and S. I. Choi, Water Sci. Technol., 1997, 36, 69-75.

57 M. Y. Han and H. K. Lee, Colloids Surf., A, 2002, 202, $23-31$.

58 M. Mellema, J. H. J. van Opheusden and T. van Vliet, J. Chem. Phys., 1999, 111, 6129-6135.

59 A. Ashkin, Phys. Rev. Lett., 1970, 24, 156-159.

60 A. Ashkin, J. M. Dziedzic, J. E. Brorkholm and S. Chu, Opt. Lett., 1986, 11, 288-290.

61 S. H. Xu, L. R. Lou, Y. M. Li and Z. W. Sun, Colloids Surf., A, 2005, 255, 159-163.

62 S. H. Xu, Y. M. Li, L. R. Lou and Z. W. Sun, Chin. Phys., 2005, 14, $382-385$.

63 Z. W. Sun, S. H. Xu, J. Liu, Y. M. Li, L. R. Lou and J. C. Xie, J. Chem. Phys., 2005, 122, 184904.

64 S. H. Xu and Z. W. Sun, J. Chem. Phys., 2007, 126, 144903. 